\title{
A Neoteric Optimization Methodology for Cloud Networks
}

\author{
Tayibia Bazaz \\ Department of Computer Science and Engineering, School of Engineering Science and Technology, Jamia Hamdard \\ (Hamdard University), India, New Delhi \\ Email: \{ttb.bbz@gmail.com $\}$ \\ Sherin Zafar \\ Department of Computer Science and Engineering, School of Engineering Science and Technology, Jamia Hamdard \\ (Hamdard University), New Delhi, India \\ Email: \{sherin_zafar84@yahoo.com\}
}

Received: 24 March 2018; Accepted: 10 April 2018; Published: 08 June 2018

\begin{abstract}
Cloud computing is distinctively marked by its capability of providing on demand virtualized IT resources in a pay as you go fashion. Due to its popularity, the cloud computing users are increasing day by day which has become an important challenge for cloud providers. They need to serve their users in a best possible manner. The providers should not only provide their users a secure access to resources but also need to maintain a proper balance of QOS parameters like throughput, end-to-end delay, packet delivery ratio, jitter, response time, etc. The paper proposes an approach of using a meta-heuristic algorithm called Genetic Algorithm (GA) to optimize QOS parameters like packet delivery ratio and end to end delay in cloud networks. The intelligent optimization algorithms address several shortcomings of existing protocols by improving QOS parameters in an optimum manner. The results are simulated through MATLAB based simulator and the simulated results of proposed approach exhibit optimized parameters when compared to conventional method of shortest path cloud routing approach.
\end{abstract}

Index Terms-End to End Delay, Genetic Algorithm (GA), Meta-heuristic Algorithm, Optimization, Packet Delivery Ratio, Quality of Service (QOS).

\section{INTRODUCTION}

In the present digital era of Internet, the rapid development of storage and processing technologies have made computing resources cheaper and more powerful. This trend in technology has led to the realization of a new computing model called cloud computing [1]. The peculiarity of Cloud Computing is attributable to its unique pay as you go model. Cloud computing is basically a type of internet based computing where services including delivery of software, infrastructure, and storage over the Internet are provided in an ondemand fashion and one has to pay as per his/her usage only [2]. The on-demand service provision model in Cloud computing necessitates the use of well-established Service Level Agreements (SLAs) representing a contract signed between the customer and the service provider including non-functional requirements of the service specified as Quality of Service (QOS). QOS is a prerequisite for the clients who want to connect to the servers of these service providers, in order to avail services smoothly. It can be challenging to avail services smoothly and obtain a consistent service if these servers and clients are connected just through the Internet. It is difficult to provide guarantees or even predictions of QOS in an environment where the traffic is dynamically routed through different providers [3].

Meta-heuristic algorithm like Genetic Algorithm, Ant Colony Optimization, Particle Swarm Optimization etc can be applied in this changing environment of cloud, for the optimization of QOS parameters. Metaheuristic algorithm is a high-level, problem-independent algorithmic framework that provides a set of guidelines or strategies to develop heuristic optimization algorithms. The term meta-heuristic was coined by Glover in 1986 and is derived from two Greek words, viz. Meta meaning beyond in the sense of light and heuriskein or euriskein, which means to search. Meta-heuristic algorithm is distinguished from other exact methods in a way that they do not come with a proof that optimal solution will be found within a finite amount of time. Thus, these algorithms are developed for finding a solution that is "good enough" in computing time and "small enough". Meta-heuristics have been demonstrated by the scientific community to be a superior alternative to existing traditional methods of mixed-integer optimization. Metaheuristics are often able to offer a better trade-off between solution quality and computing time especially for complicated problems or large problem instances. These algorithms can be used for different optimization problems like continuous optimization, multi-objective optimization, and stochastic optimization [4]. 
Also, security is another issue inside distributed cloud computing as the clients have no learning of their data and information stored on the cloud. This is a more pressing concern when the clients are sharing and storing their confidential and profitable data on the cloud. In these extraordinary conditions, clients can't compromise on security and hence, the cloud service providers need to ensure their clients that their data is secured and sheltered. Some of the security issues identified with the Virtual Machine are VM Sprawl, VM Escape etc. Issues related to Data Center Layer are secure information is very still, physical security of network and server etc [5].

Thus, there needs to be a proper tradeoff between QOS and security. Various security algorithms like Hash Key algorithm, MD5, AES, DES, etc can be used to prolong security in the cloud in integration with an appropriate meta-heuristic algorithm. Followed in this paper, a literature review is given in Section II, a brief description of cloud network routing in Section III and methodology in Section IV that discusses the proposed approach of the paper. Later sections include results and findings that validate the proposed approach followed by conclusions and.

\section{RELATED WORK}

P. Kaur et al. (2016) proposed a hybrid genetic algorithm approach with different population size on cloud environment and made a comparison of this proposed algorithm with the traditional Genetic Algorithm. The results of the comparison show that the new proposed algorithm is much better than the traditional one in terms of performance, availability, flexibility etc. Thus, the paper proposed a new way to improve QOS using a hybrid approach in a large scale computational environment of cloud [2]. K. Alhamzani et al. (2015) proposed and validated CLAMBS-Cross-layer Multi Cloud Application Monitoring and Benchmarking asa-Service for efficient. QOS monitoring and benchmarking of cloud applications hosted on multicloud environments. Prior to this proposed model, existing cloud-application frameworks such as Amazon CloudWatch typically monitored the entire VM as a black box. Therefore, the actual behavior of each application's component is not monitored separately [6]. B.Chitra et al. (2013) proposed work in which the problem of price and time slot mechanism between customer and service provider can be solved by the Cloud Monitoring System (CMS). Prior to this proposed work, many approaches were proposed for this problem but without taking QOS into consideration. This paper presented an approach by not only establishing Service Level Agreement through negotiation but also enhanced QOS. The monitoring can be performed by a global predicate which is a conjunction of the local properties of different elements. In the system of $\mathrm{N}$ distributed network elements, each element monitors a set of local properties. These elements are monitored by a central monitoring station which is responsible for identifying the global parameters registered in the system [7]. The paper mainly focused on enhancing the QOS by taking monitoring as one of the aspects for of increasing the Quality of Service provided by a special monitoring system [7]. T. Chen et al. (2013) proposed a decentralized architectural style for cloud-based Dynamic Data driven application systems (DDDAS), where dynamic QOS optimization is in the heart of the symbiotic adaptation. In order to reach a refined decentralized style suited for the dynamic requirements of the cloud, the architecture leverages on the classical DDDAS primitives. The paper formulated the QOS optimization problem as a dynamic multi-objective problem. Prior to this proposed architecture, there was a lack of architectural frameworks explaining the cloud requirements, which can support cloud based DDDAS [8]. K. Dasgupta et al. (2013) proposed a novel load balancing strategy using Genetic Algorithm (GA). The algorithm succeeds in balancing the load of cloud infrastructure while trying to minimize the make span of a given task set. Cloud-Analyst simulator has been used to simulate the proposed load balancing strategy. The proposed algorithm outperformed the existing approaches like First Come First Serve (FCFS), Round Robin (RR) and a local search algorithm Stochastic Hill Climbing (SHC) based on the simulation results. The results of the paper also indicated that the proposed strategy not only outperforms a few existing techniques but also guarantees the QOS requirement of a customer [9]. K. Bhatt et al. (2013) presented a comparison analysis of Particle Swarm optimization (PSO) and explanation of execution of PSO over CloudSim with graphical results [10]. K. Xiong et al. (2012) have presented an approach to study computer science performance in cloud computing. The paper discusses the relationship among the maximal number of customers, the minimal service resources and the highest level of services in order to deliver QOS guaranteed services in such a computing environment. With the help of response time they calculated what level of QOS services can be guaranteed for given service resources, then for a given number of customers, how many service resources are required to ensure that customer services can be guaranteed [11]. J. M. Pedersen et al. (2011) investigated whether latency in terms of simple ping measurements can be used as an indicator for other QOS parameters such as jitter and throughput. The results of the paper showed some correlation between latency and throughput, and between latency and jitter. But the correlation coefficients didn't bring conclusive results as these results were not consistent throughout. The results of the experiment are important to keep in mind not only while designing cloud services or architecture but also for defining QOS parameters and related SLA. Thus, the paper proposed a first step towards defining QOS parameters to be included in Service Level Agreements for cloud computing in the foreseeable future [3]. D. Aggarwal et al. (2010) proposed an optimized scheduling algorithm for cloud services. To permit maximum usage of physical resources, the paper presented a Genetic Algorithm for optimum allocation of virtual machines. The problem was first described in a mathematical way, which was further reduced to the problem of maximizing 
a function. The coding of the algorithm was done inside a load balancer or a scheduler module of the cloud environment. Thus, it optimized the resource allocation continuously and maximized the Quality of Service [12].

\section{Routing IN CLOUD NETWORK}

Routing has a significant impact on the performance of cloud computing as it is based on on-demand network. An ideal routing algorithm tries to satisfy QOS requirements which include delivery of packets during transmission within a specified time by finding optimum path. In order to search the shortest and optimum path several shortest path algorithms are introduced like breadth first algorithm, Dijkstra algorithm, etc. These algorithms can be effective in both well-defined infrastructure or infrastructure less networks as they can solve shortest path problems in polynomial time. However, for real time communications, they show high computational complexity in a dynamically changing network topologies. Shortest path problem can be defined as a technique of finding a minimal cost path including the source and destination nodes in a constantly changing environment. In these complicated solutions, neural networks and metaheuristic algorithms are promising in terms of finding an optimal solution. But these techniques may not be promising candidates in the real-time applications as they involve numerous iterations in general. Metaheuristic algorithms (like GA) and neural network techniques however, exhibit extremely fast hardware implementation and are less sensitive to network size. GA is a problem independent algorithm and tries to find an optimal solution of a problem by continuously modifying initial solution [13], [14]. Hence, in this novel approach GA has been used for routing and QOS optimization and simulation analysis discussed in Section V validates the GA based approach when compared with the traditional routing approach used in cloud network.

\section{METHODOLOGY}

After performing an intensive literature review in above Section II and analyzing routing in cloud networks, the problem of this research analysis is being identified. It is being concluded that QOS optimization in cloud is the need of the hour nowadays. It is one of the hot research areas. The main aim of this paper is to present an approach for optimization of QOS parameters viz., Packet Delivery Ratio, End to End Delay, etc. by using metaheuristic algorithm called Genetic Algorithm. GA is a type of approximation algorithm which can be used to solve optimization combinatorial problems by its global optimization property and the capability of searching multiple regions [2]. Hence, GA will be utilized in the problem of QOS based cloud performance optimization. The objective of this research work will be the use of varying number of cloudlets for enhancement of packet delivery ratio and reduction of end to end delay in a
MATLAB based simulating environment. Moreover, these results can be further validated on some cloud based simulators like CloudSim, CloudAnalyst, etc. Fig. 1 shows the steps of the proposed approach starting from taking input in terms of tasks from user and eventually leading to optimization of QOS parameters.

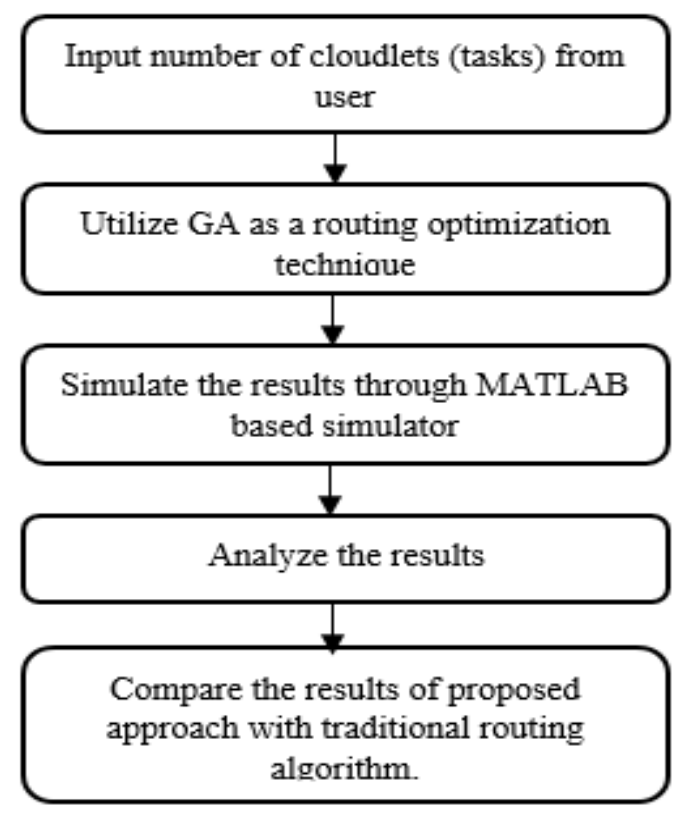

Fig.1. Methodology

Thus, the main objectives of this research study are:

- Study the traditional routing in cloud networks through CloudSim and analyze their gaps.

- Use GA as a routing optimization technique. Simulate the result through MATLAB based simulator.

- Compare the traditional routing method and the proposed GA based approach.

- Validate the proposed GA approach through optimization of various QOS parameters like packet delivery ratio, end to end delay.

\section{Algorithm for QOS optimization using GA}

Input: Required parameters (tasks) taken from the user. Output: Performs routing optimization of cloud network, hence improving Quality of Service through various parameters like packet delivery ratio and end to end delay.

- Possible path generation between source and destination

- Reiteration of the below two steps till fittest path is found.

- $\quad$ Select two possible fittest paths like P1 and P2. Perform crossover of $\mathrm{P} 1$ and $\mathrm{P} 2$, in order to obtain a new sequence path called P3.

- Mutation is done for pruning of dead nodes.

- End 


\section{RESULTS}

On the basis of literature survey and methodology, the cloudlet execution analysis was carried out in a cloud based simulator CloudSim. The analysis was based on the QOS parameters like waiting time, execution time, CPU utilization, response time by following the steps listed below:

- One data center was created with default characteristics as set by authors of CloudSim.

- The simulation was repeated three times in a row by changing the cloudlet count and the number of hosts and rest keeping all the parameters constant.

- One, two and three number of hosts were created in first, second and third iteration respectively with default configuration but having quad-core processor each.

- Also, forty virtual machines were created during simulation.

- Homogenous and heterogenous cloudlets were generated and their number was varied in each phase of simulation as shown in graphs below.

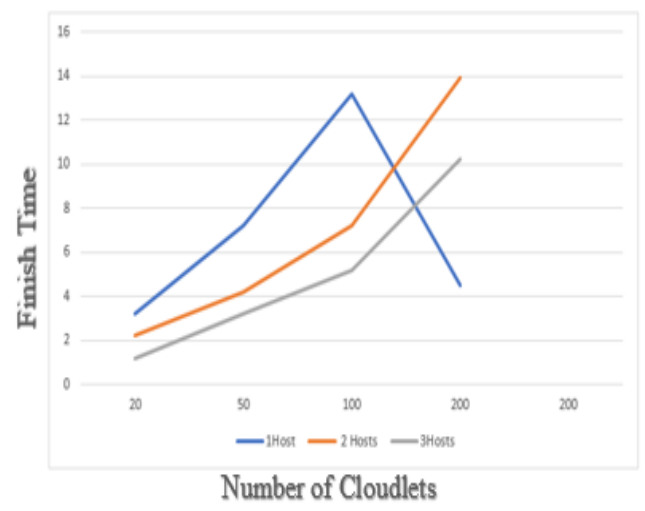

Fig.2 (a). Execution analysis of homogenous cloudlets in CloudSim

Fig. 2 (a) shows the relationship of homogenous cloudlets i.e., cloudlets with same properties and having no randomness in nature with the total number of hosts being assigned. As it is evident from Fig. 2 (a), the cloudlets execution speed increases with the increase in number of hosts.

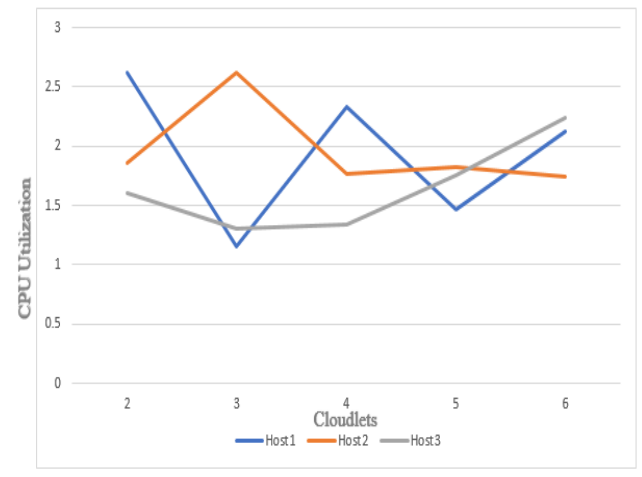

Fig.2 (b). CPU utilization analysis

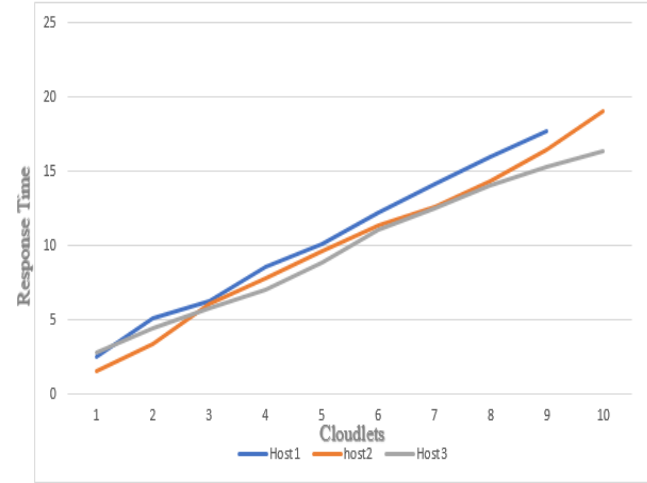

Fig. 2 (c). Response Time analysis

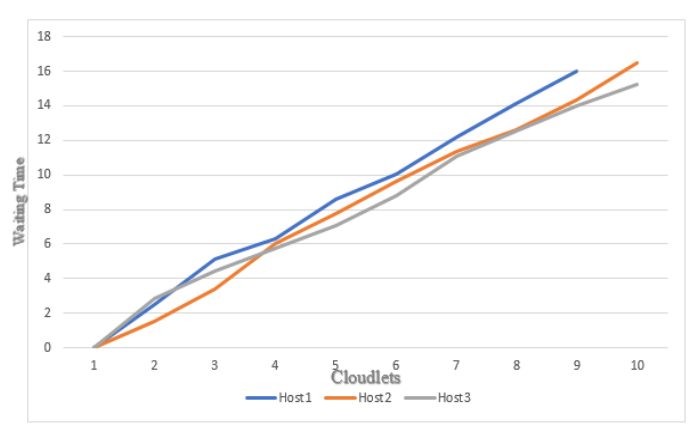

Fig.2 (d). Waiting Time analysis

In Figs. 2 (b), (c), (d), heterogenous cloudlets i.e., the cloudlets having some randomness in their nature are used with one VM and rest the configuration is same as for Fig. 2 (a).

Thus, by the analysis of cloudlets in terms of their response time, waiting time, execution time and CPU utilization with respect to VM and Hosts, it can be concluded that their properties depend on the configuration being assigned to them. Following this nature of cloudlets, their scheduling optimization can be done in terms of QOS parameters by the proposed method of using Genetic Algorithm through MATLAB based simulator.

Through the developed simulator, the proposed approach has taken the following parameters of cloudlets viz., Cloudlet Transmission Range, Cloudlet Speed, Cloudlet Data Rate and Cloudlet Traffic and has shown the following results depicted in the graphical form from Figs. 3 (a) -6 (b) below keeping cloudlet count as four (4). The proposed GA based approach has optimized two QOS parameters, Packet Delivery Ratio and End to End Delay of cloudlets i.e., optimization of cloudlet scheduling in terms of QOS on a MATLAB based simulator. Also, to enhance the analysis, the cloudlet count has been changed from four to thirteen and have displayed the results of average packet delivery ratio and average end to end delay in a tabulated form depicted in Table 1. 
Figs. 3 (a) and (b) show a comparison of packet delivery ratio and end to end delay of previous and proposed method with respect to cloudlet transmission range. The Packet Delivery Ratio is improved while a substantial reduction is observed in the End to End Delay of cloudlet transmission.

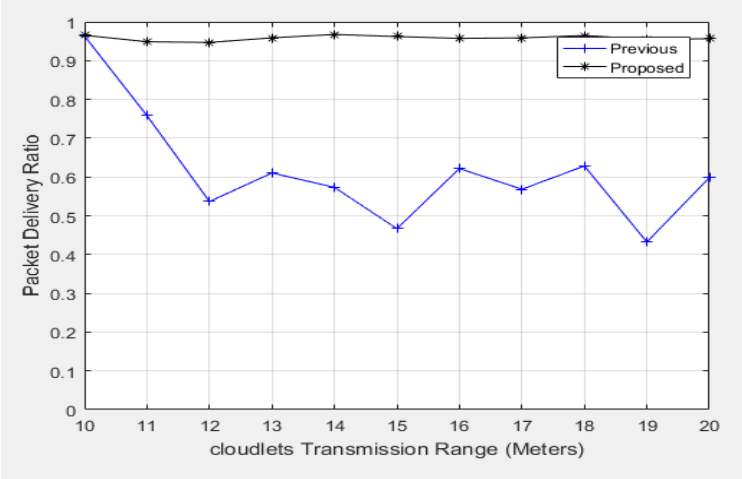

Fig.3 (a). Comparison of conventional routing technique with proposed GA based approach in terms of Packet Delivery Ratio and Cloudlet Transmission Range

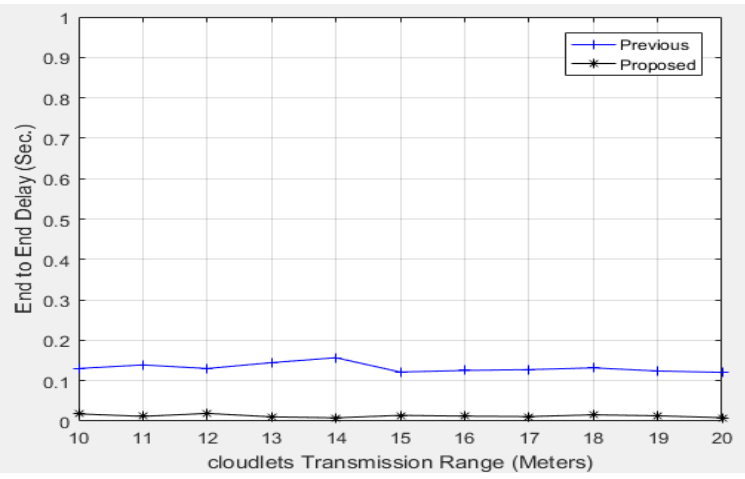

Fig.3 (b). Comparison of conventional routing technique with proposed GA based approach in terms of End to End Delay and Cloudlet Transmission Range.

Figs. 4 (a) and (b) show that by using proposed method for cloudlet scheduling in terms of Packet Delivery Ratio and End to End Delay, optimized results are obtained when compared to that of traditional method of shortest path cloud routing approach.

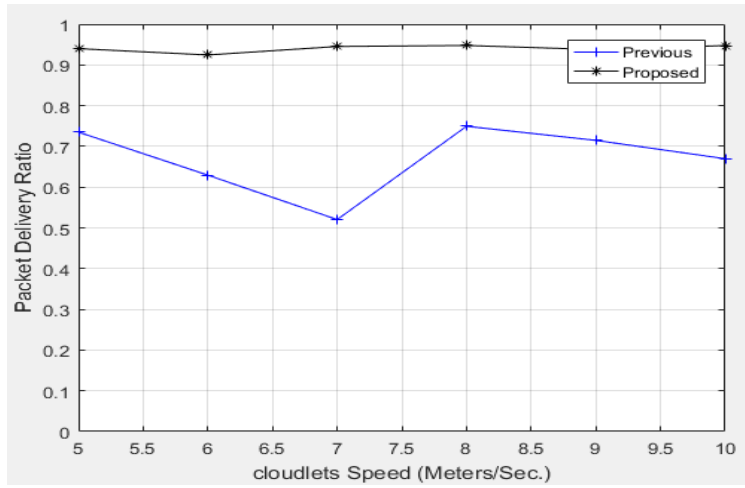

Fig.4 (a). Comparison of conventional routing technique with proposed GA based approach in terms of Packet Delivery Ratio and Cloudlet Speed.

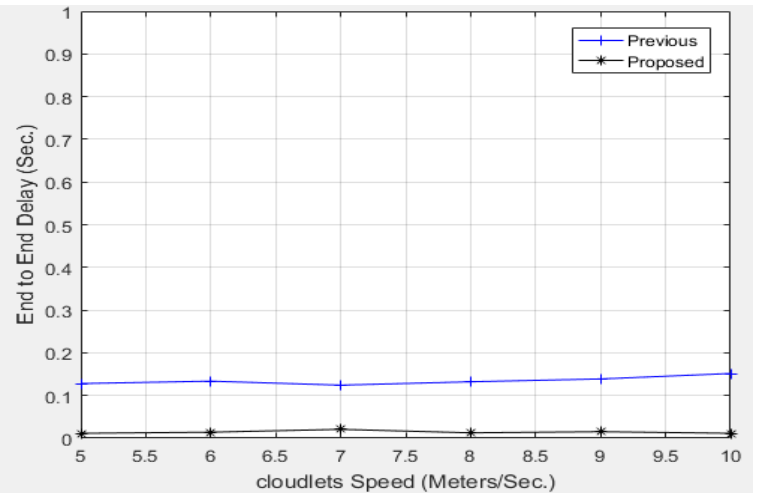

Fig.4 (b). Comparison of conventional routing technique with proposed GA based approach in terms of End to End Delay and Cloudlet Speed

In Figs. 5 (a) and (b) an analysis is made to check the cloudlet data rate with packet delivery ratio and end to end delay using previous and proposed approach. The Packet Delivery Ratio of the proposed approach is enhanced and End to End Delay is reduced with respect to the traditional routing approach.

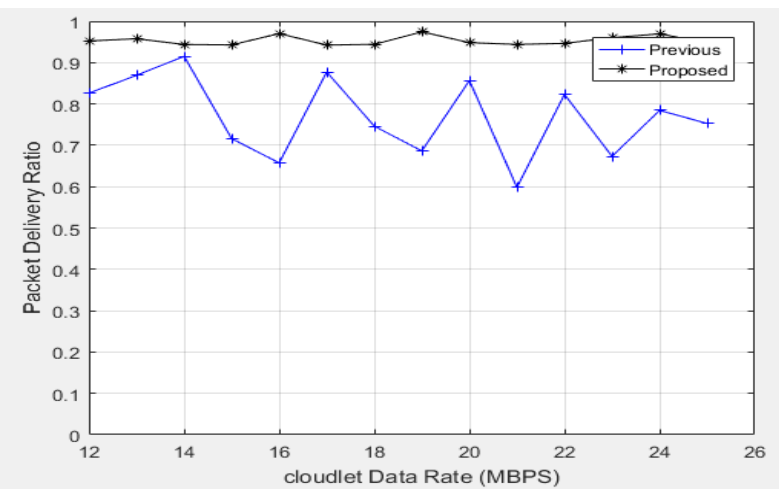

Fig.5 (a). Comparison of conventional routing technique with proposed GA based approach in terms of Packet Delivery Ratio and Cloudlet Data Rate.

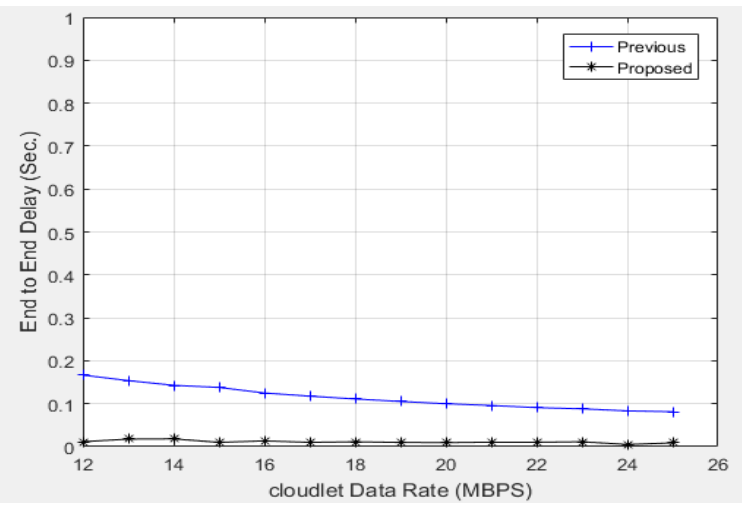

Fig.5 (b). Comparison of conventional routing technique with proposed GA based approach in terms of End to End Delay and Cloudlet Data Rate.

Figs. 6 (a) and (b) depict a comparison of shortest path routing approach with GA based approach in terms of Cloudlet Traffic with respect to End to End Delay and Packet Delivery Ratio respectively. The comparison shows optimized packet delivery ratio and end to end 
delay are obtained via GA based approach when compared to traditional routing technique.

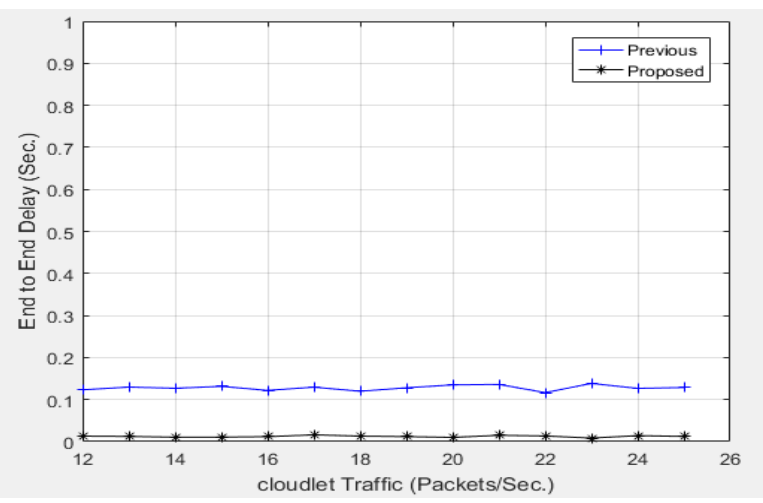

Fig.6 (a). Comparison of conventional routing technique with proposed GA based approach in terms of Packet Delivery Ratio and Cloudlet Traffic.

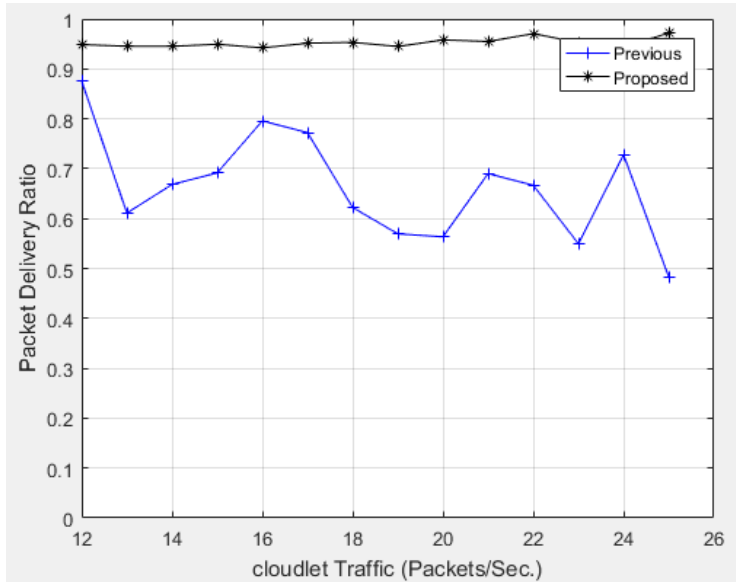

Fig.6 (b). Comparison of conventional routing technique with proposed GA based approach in terms of End to End Delay and Cloudlet Traffic.

Table 1 depicts a comparison between proposed and traditional approach by varying cloudlet count.

The results show that by using GA based approach on an average of ten (10) cloudlets from four (4) to thirteen (13), a significant improvement of $38.86 \%$ approx. is observed in average packet delivery ratio while average end to end delay has been reduced by $13.98 \%$ approx. The above simulated results are observed keeping simulation time about 100 seconds with network length and width about 100 meters. Genetic Algorithm has enhanced the execution of cloudlets almost every time during simulation. These results can be further simulated on a cloud based simulator like CloudSim using Eclipse IDE later on. The proposed approach, can be implemented in future using Example 6 of CloudSim simulator. The code of Genetic Algorithm can be integrated with the existing code of Example 6 with some appropriate changes in DataCenterBrokerClass and some other classes depending on the requirement. This setup, is expected to optimize QOS parameters along with some security enhancement in a distributed computing environment in integration with some security algorithm like AES.

\section{CONCLUSION}

Cloud networks have emerged recently as a captivating paradigm to manage and deliver resources as services over the Internet. For its proliferation amongst users, there must be a proper balance between QOS and data storage security. In this work, focus is laid on QOS optimization of cloud using Genetic Algorithm compared with shortest path routing algorithm. The proposed approach of using Genetic Algorithm optimizes the packet delivery ratio and end to end delay of cloudlet execution on a MATLAB developed simulating environment. The proposed approach uses continual search heuristics to modify the initial solution over time in order to gain the closest optimal solution as desired. Thus, helps in achieving QOS optimization. Likewise, the working of other variants of meta-heuristic algorithms will be utilized in future work analysis that will focus on optimizing results with a significant enhancement in the security of cloud. The optimized results will further be validated via a cloud based simulator like CloudSim in future and is expected to optimize the overall QOS parameters, execution time and CPU utilization of a cloudlet. Also, for better analysis of proposed work, various meta-heuristic hybrid algorithms can be used to enhance security in future which further will lead to QOS optimization in multicloud environment wherein users keep on switching from one cloud service provider to another.

Table 1. Tabular representation of cloudlet analysis

\begin{tabular}{|c|c|c|c|c|}
\hline Number of Cloudlets & $\begin{array}{c}\text { Average Packet Delivery } \\
\text { Ratio by Previous Method }\end{array}$ & $\begin{array}{c}\text { Average End To end Delay } \\
\text { by Previous Method }\end{array}$ & $\begin{array}{c}\text { Average Packet Delivery } \\
\text { Ratio by Proposed Method }\end{array}$ & $\begin{array}{c}\text { Average End To end Delay } \\
\text { by Proposed Method }\end{array}$ \\
\hline 4 & 0.58819 & 0.16146 & 0.96509 & 0.037005 \\
\hline 5 & 0.45835 & 0.14628 & 0.99398 & 0.005582 \\
\hline 6 & 0.50516 & 0.15174 & 0.9829 & 0.0043569 \\
\hline 7 & 0.61549 & 0.16915 & 0.90752 & 0.0079675 \\
\hline 8 & 0.42984 & 0.14677 & 0.92609 & 0.011516 \\
\hline 9 & 0.48426 & 0.15234 & 0.91905 & 0.029511 \\
\hline 10 & 0.80738 & 0.13199 & 0.93017 & 0.030675 \\
\hline 12 & 0.75299 & 0.14415 & 0.98287 & 0.0306879 \\
\hline 13 & 0.24896 & 0.19072 & 0.92112 & 0.0071741 \\
\hline
\end{tabular}




\section{ACKNOWLEDGMENT}

The authors wish to thank Professor Moin-ud-Din, Head of Department of Computer Science Engineering, Jamia Hamdard (Hamdard University), for his valuable support.

\section{REFERENCES}

[1] Q. Zhang, L. Cheng and R. Boutab, "Cloud computing: state-of-the art and research challenges" Journal of Internet Services and Applications, Volume 1, 2010, pp. 7-18.

[2] P. Kaur, T. Kaur "A New Era to improve the QOS on cloud using hybrid approach" International Journal Of Engineering And Computer Science, Volume 5, Issue 6, 2016, pp. 16808-16814.

[3] J. M. Pedersen, M. T. Riaz, J. C. Junior, B. Dubalski, D. Ledzinski, A. Patel "Assessing Measurements of QOS for global Cloud Computing Services" Ninth IEEE International Conference on Dependable, Autonomic and Secure Computing, 2011, pp. 682-689.

[4] F. Glover, K. Sorensen "Meta-heuristics", 2016. [Online]. Available: http://www.scholarpedia.org/article/Metaheuristics \#Definition.

[5] S. Gupta, D. S. N. Panda, D. B. Bhushan "Hash Key Based Effective Algorithm for Security in Cloud Infrastructure" International Journal of Computer Science and Technology, Volume 6, Issue 3, 2015, pp. 25-30.

[6] K. Alhamazani, R. Ranjan, P. Jayaraman, K. Mitra, F. Rabhi, D. Georgakopoulos, L. Wang "Cross-Layer MultiCloud Real-Time Application QOS Monitoring and Benchmarking As-a-Service Framework" IEEE Transactions on Cloud Computing, 2015.

[7] B. Chitra, M. Srikrishna, A. Naveenkumar "A Survey on Optimizing the QOS during Service Level Agreement in Cloud" International Journal of Emerging Technology and Advanced Engineering, Volume 3, Issue 3, 2013.

[8] T. Chen, R. Bahsoon, G. Theodoropoulos "Dynamic QOS Optimization Architecture for Cloudbased DDDAS" International Conference on Computational Science, Procedia Computer Science, 2013, pp. 1881-1890.

[9] K. Dasgupta, B. Mandal, P. Dutta, J. Mandal, S. Dam “A Genetic Algorithm (GA) based Load Balancing Strategy for Cloud Computing" International Conference on Computational Intelligence: Modeling Techniques and Applications (CIMTA), 2013, pp. 340-347.

[10] K. Bhatt, D. M. Bundele "Study and Impact of CloudSim on the run of PSO in Cloud Environment" International Journal of Innovations in Engineering and Technology, Volume 2, Issue 4, 2013, pp. 254-262.

[11] K. Xiong, H. Perros "Service Performance and Analysis in Cloud Computing", Annual SRRI Global Conference, 2012, pp. 693-700.

[12] D. Aggarwal, H. Jaiswal, I. Singh, k. Chandrasekaran "An Evolutionary Approach to Optimizing Cloud Services" Journal of Computer Engineering and Intelligent Systems, Volume 3, Issue 4, 2012, pp. 47-54.

[13] C. Wook, R. S. Ramakrishna "A Genetic Algorithm for Shortest Path Routing Problem and the Sizing of Populations" IEEE Transactions On Evolutionary Computation, Volume 6, Issue 6, 2002, pp. 566-579.
[14] P. Ponnusamy, S. Abinaya "Virtual network Routing in Cloud Computing Environment" International Conference on Computer Communication and Informatics (ICCCI), INDIA, 2016.

[15] S. Zafar, M. K. Soni, M. M. S. Beg "An Optimized Genetic Stowed Biometric Approach to Potent QOS in MANET" Procedia Computer Science 62, pp. 2015, 410418.

[16] S. Zafar, M.K. Soni "Biometric Stationed Authentication Protocol (BSAP) Inculcating MetaHeuristic Genetic Algorithm" I.J. Modern Education and Computer Science, 2014, pp. 28-35.

[17] S. Zafar, M. K. Soni "A Novel Crypt-Biometric Perception Algorithm to Protract Security in MANET" I.J. Computer Network and Information Security, 2014, pp. 64-71.

[18] S. Zafar, M. K. Soni "Secure Routing in MANET through Crypt-Biometric Technique" Proceedings of the 3rd International Conference on Frontiers of Intelligent Computing: Theory and Applications (FICTA), 2014.

[19] S. Zafar, M. K. Soni, M. M. S. Beg "QOS Optimization in Networks through Meta-Heuristic Quartered Genetic Approach" ICSCTI, IEEE, 2015.

[20] V. Singh, V. Kumar and K. Bansal "Research On Application of Perceived QOS Guarantee Through Infrastructure Specific Traffic Parameter Optimization" International Journal of Computer Network and Information Security, Volume 6, Issue 3, 2014, pp. 59-65.

[21] A. Bardsiri, S. Hashemi "QOS Metrics for Cloud Computing Services Evaluation" Intrnational Journal Of Intelligent System and Applications, Volume 6, Issue 12, 2014, pp. 27-33.

[22] T. Bazaz "Security Enhancement in Cloud Networks By Neoteric Techniques" IJARCS, Volume 8, Issue 2, 2017, pp. 29-32.

\section{Authors' Profiles}

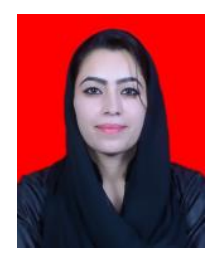

Tayibia Bazaz has pursued her M.Tech from Jamia Hamdard University, India in Computer Science Engineering and B.Tech in Information Technology Engineering from Baba Ghulam Shah Badshah University, Rajouri, J\&K in 2017 and 2015 respectively. She is a Gold Medalist of Engineering department at Jamia Hamdard. Her main interest is in optimization of Quality of Service parameters in cloud networks using meta-heuristic algorithms.

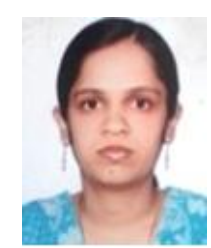

Sherin Zafar has pursued her B.Tech. and M.tech in Computer Science Engineering form RGPV Bhopal and PhD from MRIU Faridabad. She has achieved above 100 reads in Research Gate in July 2017, Faculty of Computer Science Engineering at Jamia Hamdard, New Delhi. Her main interest is in optimization in MANET, using Biometric Approach. She has published around 30 papers in SCOPUS listed journals and reviewed around 80 papers. 
How to cite this paper: Tayibia Bazaz, Sherin Zafar, " A Neoteric Optimization Methodology for Cloud Networks", International Journal of Modern Education and Computer Science(IJMECS), Vol.10, No.6, pp. 27-34, 2018.DOI: 10.5815/ijmecs.2018.06.04 